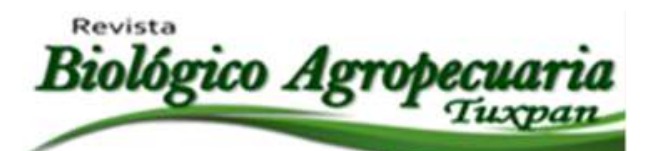

\title{
Fertilización agroquímica y orgánica en dos variedades de fresa y su respuesta en la producción de material vegetativo para propagación
}

Agrochemical and organic fertilization in two strawberry varieties and their response in the production of vegetative material for propagation

Guzmán-Castorena Alejandra ${ }^{1}$, Balandrán-Valladares Martha Irma ${ }^{1 凶}$, Porras-Flores Damian Aaron ${ }^{1}$, Luján-Aguirre Ramón Saúl ${ }^{1}$ y Cesar Arturo Berzoza-Gaytán ${ }^{1}$

${ }^{1}$ Facultad de Ciencias Agrotecnológicas. Universidad Autónoma de Chihuahua. Av. Escorza no. 900, Zona Centro. 31000 Chihuahua, Chihuahua, México

${ }^{凶}$ Autor de correspondencia: $\underline{\text { mbalandran@uach.mx }}$

Recibido: $15 / 04 / 2019$

Aceptado: 15/05/2019

\section{RESUMEN}

La fresa es un cultivo que se propaga principalmente por estolones y coranas, es por ello, la importancia de la fertilización para la obtención de material vegetativo. El objetivo de la presente investigación fue evaluar el efecto de la aplicación de dos tipos de fertilización en la producción de estolones, coronas e hijuelas para propagación vegetativa de las variedades Festival y Camino Real. El diseño experimental fue completamente al azar con dos tratamientos y un testigo, con cinco repeticiones cada uno. No se obtuvo diferencia estadísticas en la producción de estolones entre los diferentes fertilizantes, sin embargo si existe entre el testigo y los tratamientos. No existió diferencia estadística entre las variedades evaluadas en las respuestas medidas. La producción de coranas tuvo diferencias entre los tratamientos obteniendo mejor producción la fertilización agroquímica, seguida de la orgánica y finalmente el testigo. La respuesta de la planta en la producción de hijuelas fue similar al de producción de corona. El área foliar se vio beneficiado mayormente por la aplicación de fertilización agroquímica. Con la finalidad de obtener material vegetativo para propagación de fresa de las variedades Festival y Camino Real durante el periodo de agosto a diciembre se observó que para corona e hijuelas el fertilizante agroquímico fue el que promovió el desarrollo, sin embargo en lo producción de estolones puede utilizarse el fertilizante orgánico o el agroquímico.

Palabras claves: fertilización, fresa, agroquímicos, orgánicos, material vegetativo, propagación.

\begin{abstract}
Strawberry is a crop that propagates mainly by stolons and crowns, that is why fertilization is important to obtain vegetative material. The objective of the present investigation was to evaluate the effect of the
\end{abstract}


application of two types of fertilization in the production of stolons, crowns and suckers for vegetative propagation of the Festival and Camino Real varieties. The experimental design was completely randomized with two treatments and one control, with five repetitions each. There were no statistical differences in the stolon production between the different fertilizers, however, it exists between the control and the treatments. There is no statistical difference between the varieties evaluated in the measured responses. The production of crowns had differences between the treatments, being the best production, the agrochemical fertilization, then the organic production and finally the control. The response of the plant in the production of suckers was similar to that of crown production. The foliar area was benefited mainly by the application of agrochemical fertilization. In order to obtain vegetative material for strawberry propagation of the Festival and Camino Real varieties during the period of August to December it was observed that for crowns and suckers the agrochemical fertilizer was the one that promoted the growth, however in the stolons production organic or agrochemical fertilizer can be used.

Keywords: fertilization, strawberry, agrochemicals, organic, vegetative material, propagation

\section{INTRODUCCIÓN}

La fresa (Fragaria ananassa Duch.) es un cultivo que tiene buena aceptación en el mercado, lo que la coloca como una de las frutillas más importante a nivel mundial. El auge del cultivo se debe a sus propiedades características como el sabor, color, aroma y sobre todo el contenido rico en vitamina $\mathrm{C}$. Además de vitamina B1, B2, B3, B6 y E en menor cantidad (Chaves et al., 2017; Domini, 2018). En México los principales productores son Guanajuato, Michoacán, Baja California y Estado de México_(SAGARPA, 2017; SIAP, 2019). No fue sino hasta la década de los 80 's cuando se dio la introducción de este cultivo en el estado de Chihuahua con las variedades Aroma, Camarosa, Diamante, Pájaro, Seascape, Parker, Chandler y Gaviota variedades que presentaron rendimiento y producción sobresaliente para la región (Villalobos-Díaz et al., 2014).

La planta de fresa es importada principalmente de Norte América donde se encuentra hibridada de forma natural (Bonet, 2010; Domini, 2012;
Ávila-Arce y González-Milán 2012; Husaini y Neri, 2016). Al ser una planta hibrida la fresa no puede reproducirse sexualmente. Debido a esto su propagación se lleva a cabo por división de la corona o por estolones (Avitia-Garcia et al., 2014).

Sin embargo, si las condiciones no son las adecuadas para la disponibilidad de nutrientes, la planta se verá afectada en su desarrollo (Carvajal y Yahia, 2012; Mena-Chacón et al., 2017). Aunque el cultivo de fresa no presenta exigencias altas para la fertilización se debe de tomar en cuenta que es un arbusto de alta producción, por lo tanto se debe mantener programas nutrimentales adecuados para reponer la extracción de nutrientes y de esta forma mantener la fertilidad del suelo (OlmosOropeza et al., 2015; Tucuch et al., 2017). Actualmente se utilizan diferentes sustancias para mantener a la planta en un estado nutrimental adecuado, uno de ellos es el uso de agroquímicos los cuales son empleados comúnmente (Mena et al., 2017) y los abonos orgánicos productos de desechos de origen vegetal o animal, que han sufrido 
transformaciones a travéz de un proceso de descomposición (Félix et al., 2008; Cárdenas y Yaselga, 2015).

La producción de estolones por la planta de fresa es muy importante por lo tanto mantener una nutrición mineral adecuada es la base para un desarrollo sano de la planta (Tucuch-Pérez et al., 2017). Para determinar el impacto de la fertilización agroquímica y orgánica el objetivo de la presente investigación fue evaluar el efecto de la aplicación de dos diferentes tipos de fertilización en la producción de estolones, coronas e hijuelas para propagación vegetativa de las variedades Festival y Camino real.

\section{MATERIALES Y MÉTODOS}

El experimento se desarrolló en el rancho Artalejo $\quad\left(28^{\circ} 44^{\prime} 27.8^{\prime \prime} \mathrm{N} \quad 105^{\circ} 57^{\prime} 30.5^{\prime \prime} \mathrm{W}\right)$ ubicado en la Colonia los Leones en el municipio de Chihuahua, México. Las plantas de fresa fueron colocadas dentro de un microtune de 1.80 metros de alto y $5 \mathrm{~m}$ de largo con una altura de $1.35 \mathrm{~m}$ con una malla sombra de $65 \%$ de luminosidad.

El material vegetativo fue puesto de manera individual en masetas de polietileno negro de tres litros. El sustrato utilizado fue 50/50 de peat moos y arena tamizada en malla número 20. El riego fue cada tercer día aplicando un litro de agua por maceta para evitar humedad excesiva y el moho gris provocado por el hongo patógeno Botrytis cinérea (Cháves y Wang, 2004).

Se realizaron 12 aplicaciones edáficas cada 15 días con fertilizante agroquímico 20-30-10(N, P y K) y uno orgánico elaborado por humus de lombriz.

Se registraron lecturas para determinar las concentraciones de clorofila en hoja fresca por medio del aparato Minolta SPAD modelo 502. Este evalúa cuantitativamente la intensidad del verdor de la hoja (650 a $940 \mathrm{~nm}$ ) siempre a las 12:00 horas (Cunha et al., 2015; Ojeda-Barrios et al., 2016)

El diseño experimental fue completamente al azar con dos tratamientos y un testigo, con cinco repeticiones por tratamiento de las variedades festival y camino real. Se realizó un análisis ANOVA con un $\alpha=0.05$ y una comparación de medias por el método Tukey, para lo que utilizo el programa estadístico IBM SPSS Statistics 20.

\section{RESULTADOS Y DISCUSIÓN}

El desarrollo de la planta se beneficia por la aplicación de fertilizantes. Se analizó la respuesta de la planta de fresa en la producción de estolones y encontramos diferencias significativas (Figura 1), donde las medias de los tratamientos de fertilización agroquímica y orgánica son similares estadísticamente, pero ambas difieren de la producción de las plantas testigo. Obteniendo un promedio de 5 estolones por planta de la variedad Festival y 6 de la Camino real bajo aplicación del fertilizante agroquímico; 5 estolones en planta Festival y 5 Camino real en condiciones de fertilización orgánica y en lo referente a la respuesta del testigo encontramos que la variedad Festival produjo 4 estolones y la Camino Real 5, todas evaluadas durante un periodo de cinco meses. Esto concuerda con los resultados reportados por Romero-Romano et al. (2012), los cuales obtuvieron la misma respuesta al evaluar diferentes tipos de productos orgánicos y químicos donde no se encontró diferencias en la respuesta de la planta. Esto nos podría indicar que durante el periodo de agosto a diciembre en el cual se realizó la fertilización el uso de 
agroquímicos o productos orgánicos tendrá el mismo efecto en la producción de estolones. Sin embargo, durante el proceso de producción estos resultados podrían ser distintos, ya que, como lo mencionan diversos investigadores los requerimientos nutrimentales van a variar según la etapa fenológica del cultivo (Olmos-Oropeza et al., 2015; Avitia-García et al., 2014)

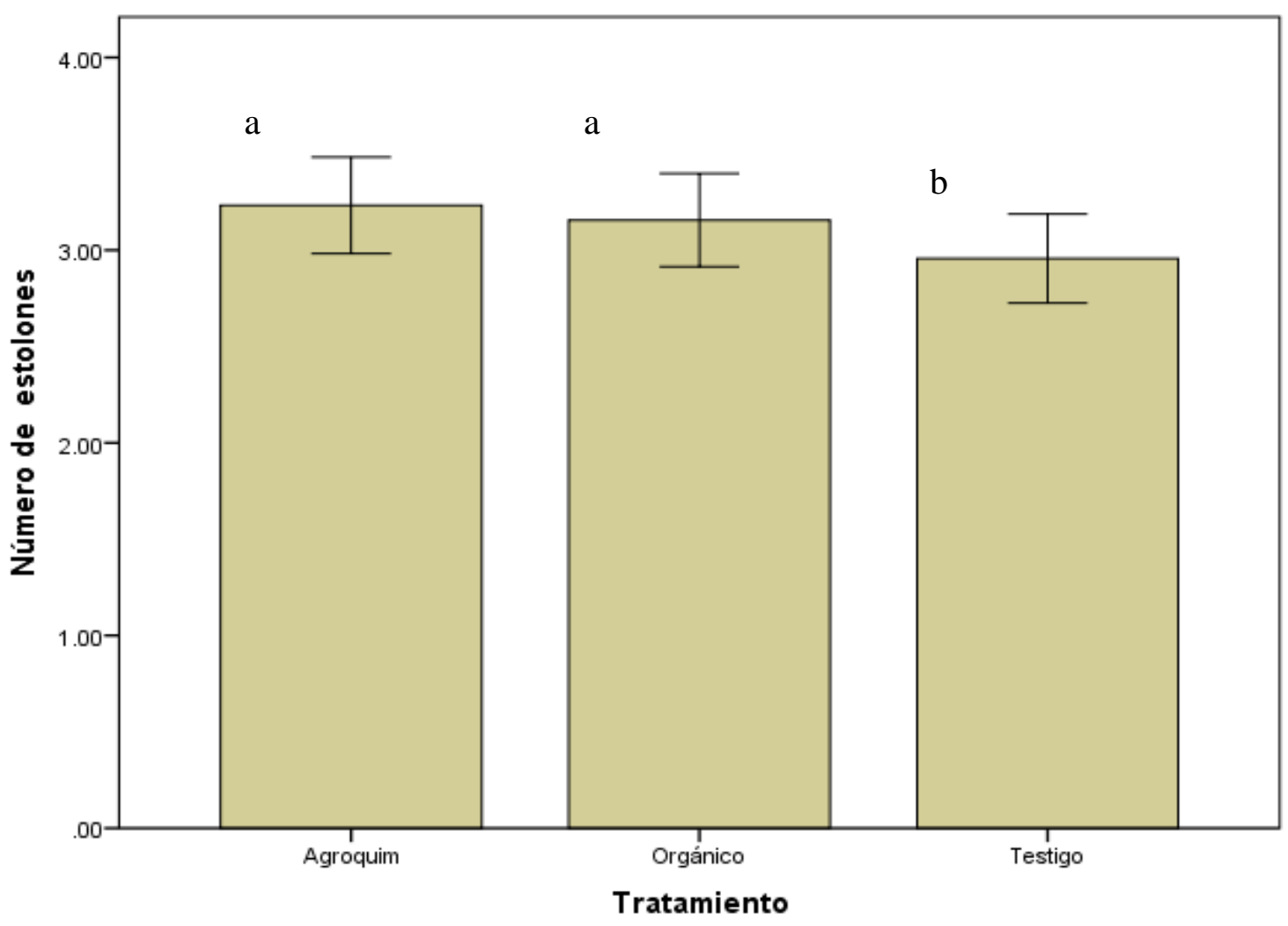

Barras de error: +/- 1 DT

Figura 1. Comportamiento de la producción de estolones por plantas madre bajo dos diferentes tipos de fertilización.

La producción de coronas por las plantas bajo dos tipos de fertilización obtuvo diferencias significativas. La producción de coronas puede variar entre variedades de fresa (RodríguezBautista et al., 2012), sin embargo, en este trabajo no se encontró diferencias estadísticas entre Festival y Camino Real. La aplicación de fertilizante agroquímico presento un mayor número de coronas producidas por planta, mientras que el testigo presentó una producción inferior a las que fueron expuestas a fertilización orgánica. De igual modo la producción de hijuelas tuvo un comportamiento similar al de las coronas (Figura 2). La mayor presencia se dio en las plantas tratadas con agroquímico, seguido del orgánico y finalmente el testigo con una menor cantidad de hijuelas por hijos (Figura 3). 


\section{Guzmán-Castorena et al., 2019}

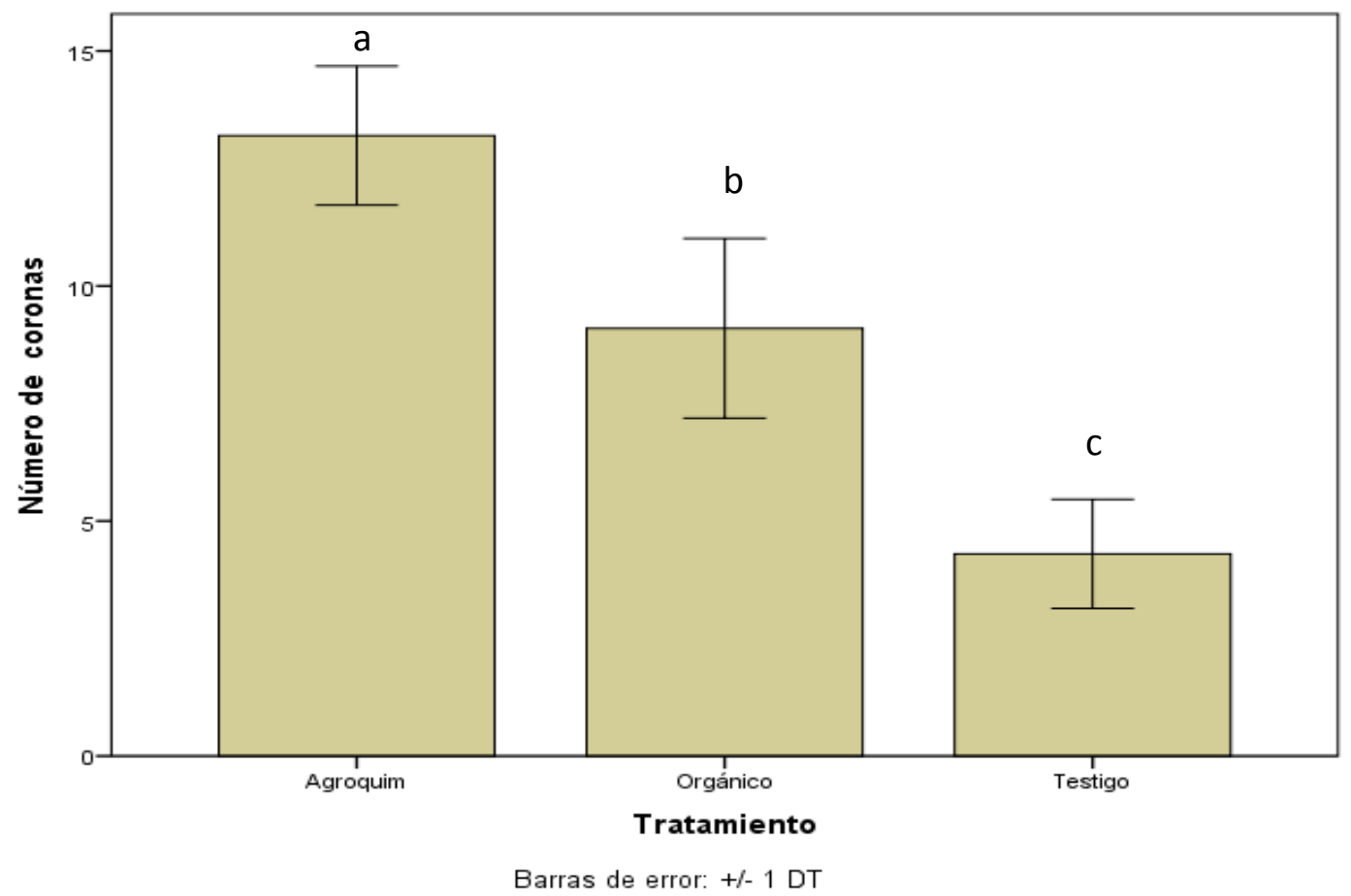

Figura 2. Comportamiento de la producción de coronas por plantas madre bajo dos diferentes tipos de fertilización.

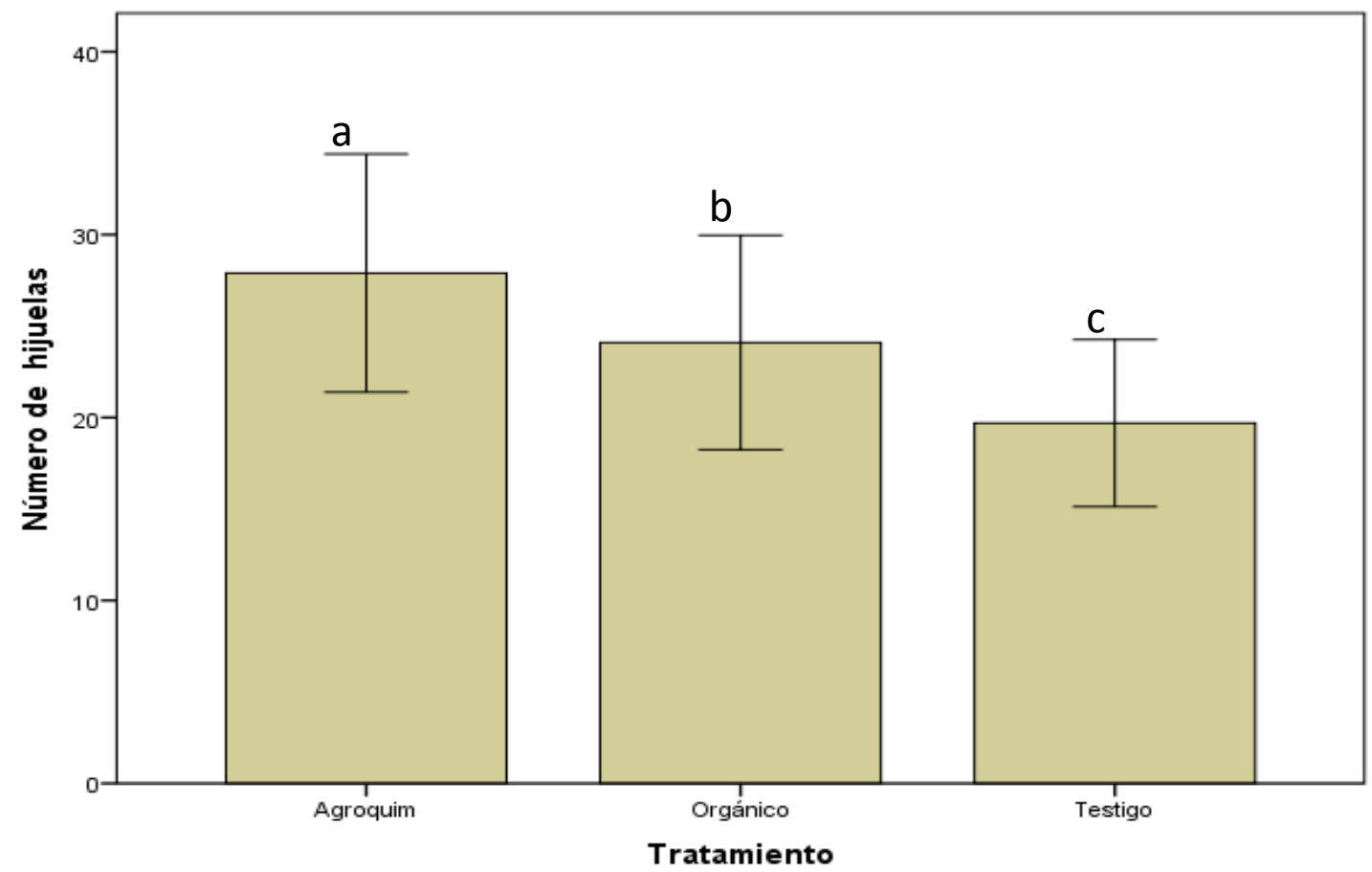

Figura 3.

Barras de error: +/- 1 DT

Figura 3. Comportamiento de la producción de hijuelas por plantas madre bajo dos diferentes tipos de fertilización 
Se tiene reportes que la fertilización orgánica mejora el área foliar (León-López et al., 2014.). El número de hojas por plantas se vio aumentado a comparación del testigo por la aplicación de los dos tratamientos. La mayor producción foliar se dio en el tratamiento del producto agroquímico (Figura 4).

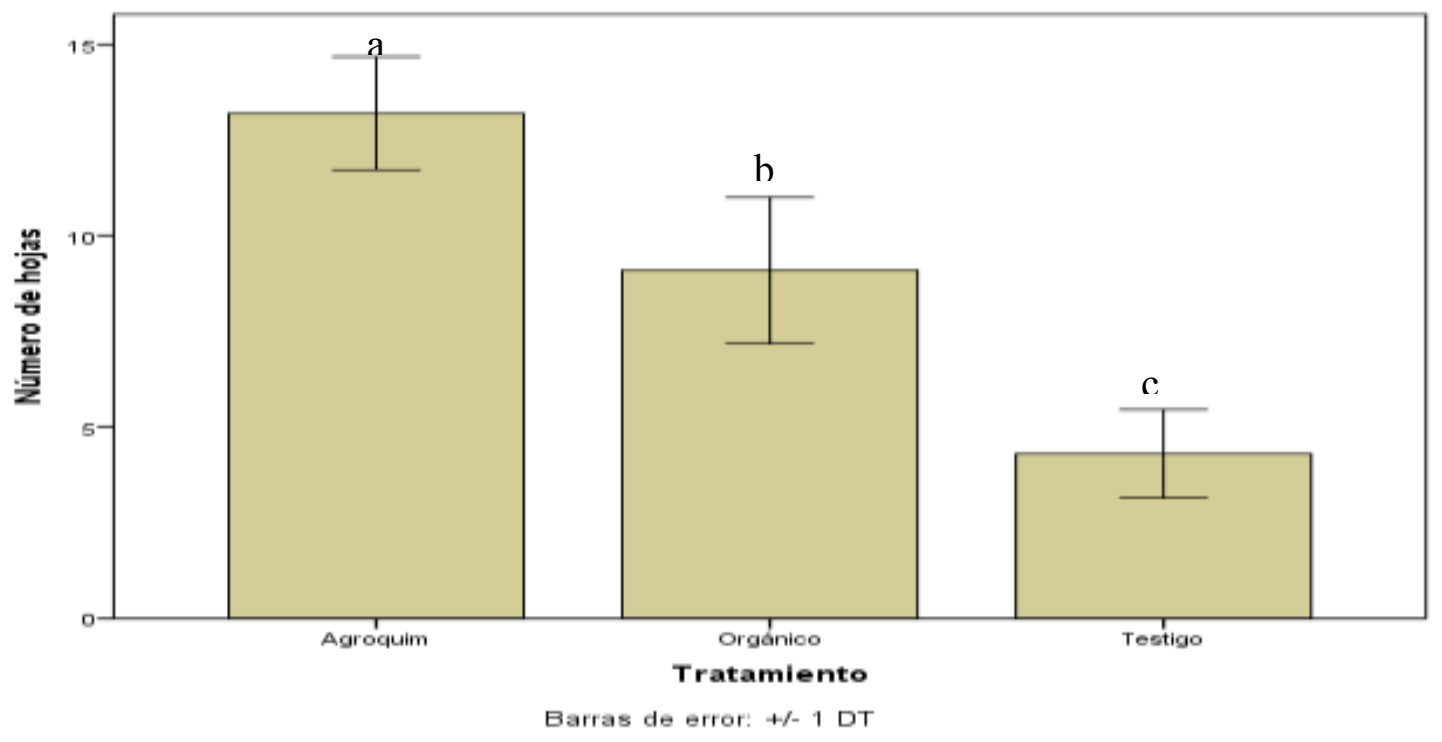

Figura 4. Comportamiento de la producción de hojas por plantas madre bajo dos diferentes tipos de fertilización.

Se elaboró un análisis de varianza para conocer el efecto de los tratamientos sobre la presencia de clorofila en las variedades evaluadas a lo largo del proceso de fertilización y no se encontró diferencias significancia alguna entre las interacciones posibles a realizarse a lo largo del tiempo. Además dentro de los resultados encontramos que se presenta una tendencia cuadrática ente los datos obtenidos por el SPAD durante el periodo de medición. Sin embargo, al realiza comparación de medias encontramos los tratamientos donde se aplicó fertilización orgánica y química muestran contenidos similares de clorofila en hoja durante los distintos tiempos de aplicación diferentes a los contenidos que se registraron para el testigo, por lo que la aplicación de fertilización favorece la presencia de clorofila en las plantas madre de estas variedades en condiciones de microtunel. Latsague et al.
(2014), menciona que el cultivo de chile se obtuvo mejores resultado en presencia de clorofila en distintos niveles de fertilización que en plantas de chile sin fertilizar.

\section{CONCLUSIÓN}

Los resultados obtenidos muestran que para el periodo de agosto diciembre se puede utilizar cualquiera de los dos tipos de fertilización evaluados si la finalidad es la formación de estolones, debido a que no se presentaron diferencias entre estos dos tratamientos. Sin embargo para la producción de coronas, hijuelas y mayor un mayor número de hojas se recomienda la aplicación de fertilizantes agroquímicos para las variedades Festival y Camino real bajo condiciones de microtunel. 


\section{LITERATURA CITADA}

Ávila-Arce, A., González-Milán D. J.. 2012. La competitividad de las fresas (Fragaria spp.) mexicanas en el mercado nacional, regional y de Estados Unidos. Agric. Soc. Desarro. 9(1):17-27.

Avitia-García, E., J., Pineda-Pineda, A. A., Castillo-González, L. I. Trejo-Téllez, T. Corona-Torres y E., Cervantes-Urbán. 2014. Extracción nutrimental en fresa (Fragaria $\mathrm{x}$ ananassa Duch.). Revista Mexicana de Ciencias Agrícolas. 5(3):519-524.

https://doi.org/10.29312/remexca.v5i3.955 Carvajal, L., Yahia, H., Cartagena, R., Peláez, C., Gaviria, C., Rojano, B. 2012. Capacidad antioxidante de dos variedades de Fragaria $\mathrm{x}$ ananassa (Weston) Duchesne (Fresa) sometidas a variaciones en la nutrición vegetal. Revista Cubana de Plantas Medicinales. 17(1):37-53.

Chaves N. y A. Wang. 2004. Combate del moho gris (Botrytis cinérea) de la fresa mediante Gliocladium roseum. Agronomía Costarricence. 28(2): 73-85.

Cunha A.R., L. Katz, A. P. Sousa y R. A. Martínez-Uribe. 2015. Índice SPAD en el crecimiento y desarrollo de plantas de lisianthus en función de diferentes dosis de nitrógeno en ambiente protegido. Idesia (Arica). 33(2): 97105. https//doi.org/10.4067/S0718-342 92015000200012

Domini, A. K. 2018. Potencialidades del quitosano para la fresa. Usos en la mejora y conservación de los frutos. Cultivos Tropicales. 39(1):134-142.

Domini, A. K. 2012. Mejora genética de la fresa (Fragaria ananassa Duch.), a través de métodos biotecnológicos. Cultivos Tropicales. 33(3):34-41

Husaini, A. y D. Neri. 2016. Strawberry Grawth, developmet and diseases. Boston, MA. ISBN 9781780646640. https://doi.org/10.1079/9781780646633.00
Jaime Alberto Félix - Herrán, J. A., R. R. del Sañudo - Torres, G. E. Rojo - Martínez, R. Martínez - Ruiz y V. Olalde Portugal. 2008. Importancia de los abonos orgánicos. Ra Ximhai. 4(1): 57-67.https://doi.org/10.35197/rx.04.01.20 Lastague, M., P. Sáez y M. Mora. 2014. Effect of the fertilization with nitrogen, phosphorus and potassium, on the foliar content of carbohydrates, proteins and photosynthetic pigments in plants of Berberidopsis corallina Hook.f. Gayana Bot. $\quad 71(1)$ : $37-42$. https://doi.org/10.4067/S0717-66432014 León López, L., D. L. A. Guzmán-Ortíz, J A. García Berumen, C. G. Chávez Marmolejo y J. J. Peña-Cabriales. Consideraciones para mejorar la competitividad de la región "El Bajío" en la producción nacional de fresa. Rev. Mex. Cienc. Agríc. 5(4): 673-686. https://doi.org/10.29312/remexca.v5i4.929 Mena-Chacón, L. M., G. J. SarmientoSarmiento y P. Camargo-Saucedo. 2017. Impacto del abonamiento integral en el rendimiento y calidad de fresa (Fragaria $\mathrm{x}$ ananassa Duch.) cv. Selva bajo sistema de riego por goteo y cobertura plástica. Scientia Agropecuaria 8(4): 357 - 366. https://doi.org/10.17268/sci.agropecu.201 Ojeda-Barrios, D. L., E. Sánchez-Chávez, J. P. Sida-Arreola, R. Valdez-Cepeda, M. Balandrán-Valladares. 2016. The impact of foliar nickel fertilization on urease activity in pecan tres. Journal of Soil Science and Plant Nutrition. 16 (1): 237 247. https://doi.org/10.4067/S0718-95162

Olmos-Oropeza, G., J. F. Martíne-Montoya, A. Gómez-González, A. Aquino-Pérez, J. Palacio-Núñez, Bravo-Vinaja A. y V. M. Ruiz-Vera. 2015. Potencial productivo y rentabilidad del cultivo de fresa (Fragaria $x$ ananassa (Weston) Duchesne) en 
Salinas, San Luis Potosí, México.

Agroproductividad. 8(4):68-72.

Rodríguez-Bautista G., G. Calderón-Zavala G., D. Jaen-Contreras y A. Curiel-Rodríguez. 2012. Capacidad de propagación y calidad de planta de variedades mexicanas y extranjeras de fresa. Revista Chapingo Serie Horticultura. 18(1): 113123.

Romero-Romero, C. O., J., Ocampo-Mendoza, E. Sandoval-Castro y J. R. Tobar-Reyes. 2012. Fertilización orgánica - mineral y orgánica en el cultivo de fresa (fragaria $\mathrm{x}$ ananasa duch.) bajo condiciones de invernadero. Ra Ximhai. 8(3): 41-49. https://doi.org/10.35197/rx.08.03.e1.2012 Secretaria de Agricultura, Ganadería, Desarrollo Rural, Pesca y Alimentación (SAGARPA). 2017. Planeación Agrícola Nacional 2017-2030.Fresa Mexicana. Primera Edición. Mexico.

Servicio de Información Agroalimentaria y $\begin{array}{lll}\text { Pesquera } & \text { (SIAP). } & 2019 .\end{array}$ https://datos.gob.mx/busca/dataset/datosestadisticos-de-la-produccion-agricolagenerada-a-nivel-nacional

Tucuch-Perez M. A., A. Hernández-Pérez, L: A. Valdez-Aguilar, G. A. Pérez-Arias, J. C. García-Santiago y D. AlvaradoCarrillo. 2017. Aplicaciones de aluminio mantienen el crecimiento de fresa (Fragaria $x$ ananassa Duch,.) suplementada con roca fosfórica en condiciones de cultivo sin suelo. Terra Latinoamericana. 35:193-201. https://doi.org/10.28940/terra.v35i3.190

Villalobos-Díaz J., M. Basurto-Sotelo, R. Pérez-Leal, J. M. Soto-Parra y C.B. Manjarrez-Domínguez. 2014. Calidad y producción de cinco variedades de fresa (Fragaria ananassa Duch.) bajo diferentes condiciones climáticas en el estado de Chihuahua. Revista Biológico Agropecuaria de Tuxpan. 2(3):232-236.

Copyright (c) 2019 Alejandra Guzm án Castorena, Martha Irm a Balandrán Valladares, Damian Aaron Porras Flores, Ram ón Saúl Luján Aguirre y César Arturo Berzoza Gaytán

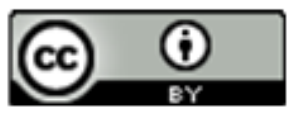

Este texto está protegido por una licencia licencia CreativeCommons 4.0 .

Usted es libre para Compartir — copiar y redistribuir el material en cualquier medio o formato-y Adaptar el documento — remezclar, transformar y crear a partir del material- para cualquier propósito, incluso para fines com erciales, siempre que cumpla la condición de:

Atribución: Usted debe dar crédito a la obra original de manera adecuada, proporcionar un enlace a la licencia, e in dicar si se han realizado cambios. Puede hacerlo en cualquier forma razonable, pero no de forma tal que sugiera que tiene el apoyo del licenciante o lo recibe por el uso que hace de la obra.

Resumendelicencia - Textocompletodelalicencia 